\title{
EQUIVARIANT BP-COHOMOLOGY FOR FINITE GROUPS
}

\author{
N. YAGITA
}

Dedicated to Professor T. Yamanoshita on the occasion of his 60 th birthday

\begin{abstract}
The Brown-Peterson cohomology rings of classifying spaces of finite groups are studied, considering relations to the other generalized cohomology theories. In particular, $\mathrm{BP}^{*}(M)$ are computed for minimal nonabelian $p$ groups $M$. As an application, we give a necessary condition for the existence of nonabelian $p$-subgroups of compact Lie groups.
\end{abstract}

\section{INTRODUCTION}

The topology of classifying space $B G$ for a finite group $G$ is important in algebraic topology. Given generalized cohomology theory $h^{*}(-), h^{*}(B G)$ plays the central role, e.g., cohomology of a group, completion of the representation ring and the Burnside ring when $h$ is the ordinary cohomology, the complex $K$ theory, and the stable cohomotopy theory, respectively. Recently, the Morava $K$-theory of $B G$ has been studied by Hopkins, Kuhn, and Ravenel [20]. For simplicity, let us denote $k^{*}(B G)$ by $k^{*}(G)$.

In this paper, we study the Brown-Peterson cohomology $\mathrm{BP}^{*}(G)$ for a prime $p$ and the related cohomology $k^{*}(G)$ with the coefficient $k^{*}=\mathrm{BP}^{*} /(\operatorname{Ideal} S)$, where $S$ is a set of generators in $\mathrm{BP}^{*}$.

Landweber showed [3] that $\mathrm{BP}^{*}\left(Z / p^{r}\right)$ is a flat $\mathrm{BP}^{*}$-module and for an abelian group $A, \mathrm{BP}^{*}(A)$ is given by the tensor product of $\mathrm{BP}^{*}\left(Z / p^{r}\right)$. For nonabelian $p$-groups, when $|G|=p^{3}, \operatorname{BP}^{*}(G)$ is determined by Tezuka-Yagita [11] and some relations to the other cohomology theories are given by $\operatorname{BP}^{*}(G)$ $\otimes_{\mathrm{BP}^{*}} Z_{(p)}=H^{\text {even }}(G)$ and $K(n)^{*}(G)=K(n)^{*} \otimes_{\mathrm{BP}^{*}} \mathrm{BP}^{*}(G)$.

Consider the map induced from restrictions

$$
r: k^{*}(G) \rightarrow \operatorname{Liminv} k^{*}(A),
$$

$A \subset G$, conjugacy classes of abelian groups. Ravenel conjectured that for $k=\mathrm{BP}, r$ is an isomorphism [8]. Unfortunately, this does not hold, however, we show that for $k=\mathrm{BP}(-; Z / p), r$ is an $F$-isomorphism by using Quillen's argument, which showed that $F$-isomorphy for $k=H Z / p$, the ordinary $\bmod p$ cohomology [6]. Moreover, we show that $\rho: \mathrm{BP}^{*}(G)_{\mathrm{BP}^{*}} Z / p \rightarrow H^{*}(G ; Z / p)$ is $F$-epic.

Received by the editors December 28, 1987.

1980 Mathematics Subject Classification (1985 Revision). Primary 55N22; Secondary 57R77.

Key words and phrases. Classifying space $B G$, cohomology of groups, BP-theory. 
We will compute $\mathrm{BP}^{*}(M)$ for $M$, minimal nonabelian $p$-groups. Then $\mathrm{BP}^{*}(M)$ is a flat $\mathrm{BP}^{*}$-module and the map $r$ is injective for $k^{*}=\mathrm{BP}^{*}$. Moreover, if $G$ is a group whose $p$-Sylow subgroup is a direct product of minimal nonabelian $p$-groups and abelian groups, then $r$ is injective and $\operatorname{BP}^{*}(G) \otimes_{\mathrm{BP}^{*}}$ $P(n)^{*} \cong P(n)^{*}(G), \mathrm{BP}^{*}(G) \otimes_{\mathrm{BP}^{*}} K(n) \cong K(n)^{*}(G)$.

In the last section, to see that $\mathrm{BP}^{*}(G)$ is useful, we will study the existence of nonabelian $p$-subgroups of compact Lie groups. For example, we prove that if $G$ is a compact Lie group such that $H^{*}(G)_{(p)}=\bigwedge\left(x_{1}, \ldots, x_{n}\right)$ and $G$ contains nonabelian $p$-groups as subgroups, then $p$ divides $\left|x_{i}\right|+1$ for some $i$.

This work builds on the joint work with M. Tezuka and a letter from D. Ravenel. The author thanks them.

\section{COHOMOLOGY THEORIES}

Let $\mathrm{BP}^{*}(-)$ be the Brown-Peterson cohomology theory with the coefficient $\mathrm{BP}^{*}=Z_{(p)}\left[v_{1}, \ldots\right],\left|v_{i}\right|=-2 p^{i}+2$ for a prime $p$. Given a set of generators $S=\left(v_{i 1}, \ldots, v_{i n}, \ldots\right)$, (here $\left.v_{0}=p\right)$, by using Baas-Sullivan theory [2, 13], we can construct cohomology theory $\operatorname{BP}(S)^{*}(-)$ with the coefficient

$$
\begin{aligned}
\operatorname{BP}(S)^{*}=\mathrm{BP}^{*} /(\operatorname{Ideal} S) & =Z_{(p)}\left[v_{j} \mid j \neq i_{k}\right] \quad \text { if } p \notin S, \\
& =Z / p\left[v_{j} \mid j \neq i_{k}\right] \quad \text { if } p \in S .
\end{aligned}
$$

The cohomology $\operatorname{BP}(S)^{*}(-)$ has a good multiplication, and if $p \geq 3$ it is commutative. A useful result of this theory is the following Sullivan-Bockstein exact sequence; that is, if $v_{n}$ is not contained in $S$, then

$$
\mathrm{BP}(S)^{*}(X) \stackrel{v_{n}}{\rightarrow} \mathrm{BP}(S)^{*}(X) \stackrel{\rho}{\rightarrow} \mathrm{BP}\left(S, v_{n}\right)^{*}(X) \stackrel{\delta}{\rightarrow} \mathrm{BP}(S)^{*+2 p^{n}-1}(x)
$$

is exact, where $v_{n}$ is a map of multiplying by $v_{n}, \rho$ is the natural induced map, and $\delta$ is the $v_{n}$-Bockstein boundary map (for details see $[2,13]$ ).

The examples of $\mathrm{BP}(S)^{*}(-)$ are

$$
\begin{aligned}
P(n)^{*} & =\operatorname{BP}\left(p, v_{1}, \ldots, v_{n-1}\right)^{*}=Z / p\left[v_{n}, v_{n+1}, \ldots\right], \\
k(n)^{*} & =\operatorname{BP}\left(p, \ldots, v_{n}, \ldots\right)^{*}=Z / p\left[v_{n}\right], \quad K(n)^{*}=\left[v_{n}^{-1}\right] \cdot k(n)^{*}, \\
\operatorname{BP}\langle n\rangle & =\operatorname{BP}\left(v_{n+1}, \ldots\right)^{*}=Z_{(p)}\left[v_{1}, \ldots, v_{n}\right], \\
H Z_{(p)}^{*} & =\operatorname{BP}\left(v_{1}, \ldots\right)^{*}=Z_{(p)}, \quad H Z / p^{*}=Z / p .
\end{aligned}
$$

In this paper we consider these cohomology theories $\mathrm{BP}(S)^{*}(-)$. For simplicity of notation, we write it as $k^{*}(-)$ and denote by $\#(k)$ the cardinal number of the set $\left(p, v_{1}, \ldots\right)-S$; that is, \# $(k)=n$ if $k^{*}=Z / p\left[v_{i 1}, \ldots, v_{i n}\right]$ or $k^{*}=Z_{(p)}\left[v_{i 1}, \ldots, v_{i n-1}\right]$.

Let $G$ be a compact Lie group and $B G$ be its classifying space. Let $P G$ be a contractible free $G$-space. Then the equivariant cohomology of a $G$-space $X$ is defined by

$$
K_{G}^{*}(X)=k^{*}\left(P G \times_{G} X\right) \text { and } k_{G}^{*}(p t)=k_{G}^{*}=k^{*}(G)=k^{*}(B G) .
$$




\section{COHOMOLOGY OF ABELIAN GROUPS}

Consider the homomorphism

$$
m: S^{1} \times S^{1} \rightarrow S^{1}
$$

defined by $m(x, y)=x+y$ identifying $S^{1}=R / Z$. The induced map of classifying spaces

$$
m: B S^{1} \times B S^{1} \rightarrow B S^{1}, \quad B S^{1} \cong C P^{\infty},
$$

is the usual product map induced from the tensor bundle. Take two-dimensional elements $u, u_{1}, u_{2}$ so that $k^{*}\left(C P^{\infty} \times C P^{\infty}\right) \cong k^{*}\left[\left[u_{1}, u_{2}\right]\right]$ and $k^{*}\left(C P^{\infty}\right) \cong$ $k^{*}[[u]]$. Then the map from $(2.1)$

$$
m^{*}(u)=\sum a_{i j} u_{1}^{i} u_{2}^{j}=u_{1}+_{k} u_{2}
$$

defines the formal group law $[2,7]$.

The formal group law for $\mathrm{BP}^{*}$-theory is the universal group law for group laws over rings which are $Z_{(p)}$-modules. It is well known that

$$
\begin{aligned}
& u_{1}+{ }_{\mathrm{BP}} u_{2}=u_{1}+u_{2}+v_{1} \sum_{0<i<p} \frac{1}{p}\left(\begin{array}{c}
p \\
i
\end{array}\right) u_{1}^{i} u_{2}^{p-i}+\cdots, \\
& {[p](u)=p u+v_{1} u^{p}+\cdots+v_{n} u^{p^{n}}+\cdots}
\end{aligned}
$$

where $[p](u)$ is the $p$ th sum $u+{ }_{\mathrm{BP}} \cdots+{ }_{\mathrm{BP}} u$. Given an $m \times m$-matrix $C=\left(c_{i j}\right)$ over $Z$, it induces a map

$$
\begin{aligned}
& C: S^{1} \times \cdots \times S^{1} \rightarrow S^{1} \times \cdots \times S^{1}, \\
& C^{*}: k^{*}\left[\left[u_{1}, \ldots, u_{n}\right]\right] \rightarrow k^{*}\left[\left[u_{1}, \ldots, u_{n}\right]\right], \text { and } \\
& C^{*}\left(u_{i}\right)=\sum_{k}\left[c_{j i}\right]\left(u_{j}\right) .
\end{aligned}
$$

In particular, the short exact sequence

$$
0 \rightarrow Z / p^{r} \rightarrow S^{1} \rightarrow S^{1} \rightarrow 0
$$

induces the map of fiber spaces $S^{1} \rightarrow B Z / p^{r} \rightarrow B S^{1}$. This follows the Gysin exact sequence and we have

$$
k^{*}\left(Z / p^{r}\right) \cong k^{*}[[u]] /\left[p^{r}\right](u) .
$$

Landweber showed that the Künneth formula holds for $\mathrm{BP}^{*}$-cohomology of abelian groups; that is,

$$
\begin{aligned}
\mathrm{BP}^{*}\left(\bigoplus^{s} Z / p^{r_{i}}\right) & \cong \bigotimes_{\mathrm{BP}^{*}}^{s} \mathrm{BP}^{*}\left(Z / p^{r_{i}}\right) \\
& \cong \mathrm{BP}^{*}\left[\left[u_{1}, \ldots, u_{s}\right]\right] /\left(\left[p^{r_{1}}\right]\left(u_{1}\right), \ldots,\left[p^{r_{s}}\right]\left(u_{s}\right)\right) .
\end{aligned}
$$

We always assume that $\otimes$ means the complete tensor product. Of course, the Künneth formula of this type does not hold for $k=H Z(p)$. By using Stretch's argument [10], we prove the Künneth formula for smaller $p$-rank groups. 
Lemma 2.7. Let $A$ be an abelian p-group with $\operatorname{rank}_{p} A \leq \#(k)$. Then $k^{*}(A) \cong$ $\bigotimes_{k^{*}} k^{*}\left(Z / p^{r_{i}}\right)$.

Proof. From the Sullivan-Bockstein exact sequence and (2.6), it is easily seen that $\rho: \mathrm{BP}^{*}(A) \rightarrow k^{*}(A)$ is epic if and only if $k^{*}(A) \cong \otimes_{k^{*}} k^{*}\left(Z / p^{r}\right)$. Hence we need only prove the result for the case $\operatorname{rank}_{p} A=\#(k)$.

Let $\operatorname{rank}_{p} A=n$ and $k^{*}=Z / p\left[v_{i 1}, \ldots, v_{i n}\right]$. Let us write $s_{i}=\left[p^{r_{i}}\right]\left(u_{i}\right)$, $S_{n}=\left(s_{1}, \ldots, s_{n}\right)$, and $k^{*}\left[U_{n}\right]=k^{*}\left[\left[u_{1}, \ldots, u_{n}\right]\right]$. Then we need to prove that $S_{n}$ is regular in $k^{*}\left[U_{n}\right]$. Assume by induction that $S_{n-1}$ is regular in $k^{\prime *}\left[U_{n-1}\right]$ for all $k^{\prime}$ with $\#\left(k^{\prime}\right)=n-1$ and $k^{\prime 0}=Z / p$.

Suppose the regularity does not hold, namely, there is $a \in k^{*}\left[U_{n}\right]$ such that $a s_{n} \in$ Ideal $S_{n-1}$ but $a \notin \operatorname{Ideal} S_{n-1}$. Let us write

$$
s_{n}=v_{i 1}^{l} u_{n}^{l^{\prime}}+\cdots \quad \text { and } a=a_{1} u_{n}^{j}+a_{2} u_{n}^{j+1}+\cdots
$$

with $a_{1} \notin \operatorname{Ideal} S_{n-1}$. Since

$$
a s_{n}=a_{1} v_{i 1}^{l} u_{n}^{l^{\prime \prime}}+a u_{n}^{l^{\prime \prime}+1}+\cdots \in \operatorname{Ideal} S_{n-1},
$$

we have $a_{1} v_{i 1}^{l} \in \operatorname{Ideal} S_{n-1}$. Therefore there is $b \in k^{*}\left[U_{n-1}\right]$ such that $b \notin$ Ideal $S_{n-1}$ but $v_{i 1} b \in \operatorname{Ideal} S_{n-1}$.

Let $k^{\prime *}=Z / p\left[v_{i 2}, \ldots, v_{i n}\right]$. Then by the inductive assumption, $S_{n-1}$ is regular in $k^{\prime}\left[U_{n-1}\right]$. Therefore $K=k^{\prime *}\left[U_{n-1}\right] \otimes \wedge\left(e_{1}, \ldots, e_{n-1}\right), d e_{i}=s_{i}$, is a Koszul complex and it is an acyclic complex. Let us write $v_{i 1} b=\sum^{n-1} \mu_{i} s_{i}$. Then $d\left(\sum \mu_{i} e_{i}\right)=0$ in $K$ because $v_{i 1}=0$ in $k^{\prime *}$. By the exactness of $K$, we can take $c_{i j} \in k^{\prime}\left[U_{n-1}\right]$ with

$$
\sum \mu_{i} e_{i}=d\left(\sum c_{i j} e_{i} e_{j}\right)
$$

Hence we get in $k^{*}\left[U_{n}\right]$

$$
\begin{gathered}
\mu_{i}=\sum c_{i j} s_{j}+v_{i 1} l_{i}, \quad c_{i j}=-c_{i j}, \\
v_{i 1} b=\sum c_{i j} s_{i} s_{j}+\sum v_{i 1} l_{i} s_{i}=\sum v_{i 1} l_{i} s_{i} .
\end{gathered}
$$

Therefore $b=\sum l_{i} s_{i} \in \operatorname{Ideal} S_{n-1}$. This is a contradiction. Hence we prove the theorem when $k^{0}=Z / p$.

When $k^{0}=Z_{(p)}$, we can prove the theorem by similar arguments, taking $s_{i}=p^{r_{i}} u+\cdots$ and $k^{\prime *}=Z / p\left[v_{i 1}, \ldots, v_{i n-1}\right]$. Q.E.D.

Remark 2.8. For $k^{*}=k(n)^{*}$, we can easily see that

$$
k(n)^{*}(Z / p \oplus Z / p) \cong k(n)^{*}\left[\left[y_{1}, y_{2}\right]\right] /\left([p]\left(y_{1}\right),[p]\left(y_{2}\right)\right) \oplus Z / p\left[y_{1}, y_{2}\right] \alpha
$$

where $\rho(\alpha)=Q_{n}\left(x_{1} x_{2}\right)$ in $H^{*}(Z / p \oplus Z / p ; Z / p)$ and $Q_{i}$ is the Milnor exterior operation, $Q_{0}\left(x_{i}\right)=y_{i}$. Moreover, when $\#(k)=n$, we see that $k^{*}\left(\bigoplus^{n+1} Z / p\right)$ 
$\not \approx \bigotimes_{k^{*}} k^{*}(Z / p)$ because there is an element $\alpha$ such that $Q_{i 1} \cdots Q_{i n}\left(x_{1} \cdots x_{n+1}\right)$ $=\rho(\alpha)$ or $Q_{i 1} \cdots Q_{i n-1} Q_{0}\left(x_{1} \cdots x_{n+1}\right)=\rho(\alpha)$.

\section{THE RESTRICTION HOMOMORPHISM}

Restriction map $i_{A}^{*}: k^{*}(G) \rightarrow k^{*}(A)$ for all conjugacy classes of abelian subgroups $A$ of $G$ induce the map

$$
r: k^{*}(G) \rightarrow \operatorname{Liminv} k^{*}(A),
$$

$A \subset G$, conjugacy classes of abelian $p$-groups. We will show that (3.1) is an $F$-isomorphism if $\#(k) \geq \operatorname{rank}_{p} G$ and $k^{0}=Z / p$. A ring homomorphism $f: A \rightarrow B$ is said to be an $F$-isomorphism if $\operatorname{Ker} f \subset \sqrt{0}$ (nilpotent elements) and for all $b \in B$ there is $i$ such that $b^{p^{i}} \in$ Image $f$. Quillen proved the $F$-isomorphism of $r$ for $k=H Z / p$ and the conjugacy classes of elementary abelian $p$-groups $e A$ [6]. However, $\operatorname{Ker} i^{*}$ of the restriction map $i^{*}: k^{*}\left(Z / p^{2}\right) \rightarrow k^{*}(Z / p)$ is not nilpotent for $\#(k) \geq 2$, and we consider all abelian $p$-groups. Most arguments of this section are $k^{*}$-theory versions of Quillen's arguments [5].

Lemma 3.2. Let $X$ be a compact manifold and $G$ act on $X$ smoothly. If $u \in k_{G}^{*}(X)$ restricts to zero on each orbit of $X$, then $u$ is nilpotent.

Proof. The $k^{*}$-theory version of Lemma 3.9 in [5].

Theorem 3.3. The kernel of $r$ in (3.1) is nilpotent.

Proof. Let $\rho: G \hookrightarrow U$ be a unitary representation and $T$ be a maximal torus of $U$. Consider the map of equivariant cohomologies

$$
k^{*}(G) \cong k_{G}^{*}(p t) \underset{p r^{*}}{\longrightarrow} k_{G}^{*}(U / T) \underset{i^{*}}{\rightarrow} k_{G}^{*}(G x) \cong k_{G}^{*}(G / A) \cong k^{*}(A) .
$$

The orbits of $G$ on the flag manifold $U / T$ are of the form $G / A$ where $A$ is an abelian group as it is conjugated in $U$ to a subgroup of $T$.

Assume that $u \in k^{*}(G)$ and $u \mid A=0$. The image $\operatorname{pr}^{*}(u)$ restricts to zero on each orbit of $U / T$, hence it is nilpotent by Lemma 3.2. But the map $p r^{*}$ is injective. Indeed, since $k^{*}(-)$ is complex oriented, the Leray-Hirsch theorem holds; that is, $k^{*}(B G) \rightarrow k^{*}\left(P\left(\rho^{*} \xi\right)\right)$ is injective where $P\left(\rho^{*} \xi\right)$ is a $U / S^{1}$ bundle induced from $B \rho: B G \rightarrow B U$ of the universal bundle $\xi$. Q.E.D.

Let $H$ be a subgroup of $G$ such that $[G ; H]=m$. Let $\Sigma^{m}$ be the symmetric group of $m$ letters. Then there is the inclusion

$$
\Phi: G \hookrightarrow \Sigma^{m} \imath H=\Sigma^{m} \ltimes(H \times \cdots \times H) .
$$

Consider the inclusion map of classifying spaces

$$
B i:(B H)^{m} \hookrightarrow P \Sigma^{m} \times_{\Sigma^{m}}(B H)^{m}=B\left(\Sigma^{m} \ltimes H\right) .
$$

Denote by $i$, the Gysin map of $B i$, constructed by Quillen in [7] and for $k=\mathrm{BP}(S)$ in [13].

$$
i_{!}: k^{*}\left((B H)^{m}\right) \rightarrow k^{*}\left(B\left(\Sigma^{m} \ltimes H\right)\right) .
$$


Remark 3.8. Let $B G^{N}$ be an $N$-dimensional skeleton of $B G$. By dimensional reason of the spectral sequence $H^{*}\left(B G^{n}, k^{*}\right) \Rightarrow k^{*}\left(B G^{N}\right)$, we can easily prove

$$
\operatorname{Lim}_{N \rightarrow \infty} k^{*}\left(B G^{N}\right)=k^{*}(B G) .
$$

Here $B G^{N}$ is a finite complex since $G$ is a finite group. The Gysin map defined above is defined only on finite complexes; however, we can extend this to $B G$ by (3.9).

Define the Evens norm $N[H \hookrightarrow G]: k^{*}(H) \rightarrow k(G)$ by

$$
N[H \hookrightarrow G](x)=\Phi^{*}\left(i_{1} x^{m}\right) .
$$

Then we can show that this norm has the following properties by the arguments of Evens for $k^{*}=H Z_{(p)}$ in $\S 6$ in [1], namely transitivity, naturality, multiplicative property, and double coset formula.

Lemma 3.11 ((2.1) in [5]). If $u \in k^{*}\left(G^{\prime}\right)$ is such that $u \mid G^{\prime \prime}=1$ for all $G^{\prime \prime} \supsetneqq G^{\prime}$, then we have

$$
N\left[G^{\prime} \hookrightarrow G\right](u) \mid K= \begin{cases}1 & \text { if } G^{\prime} \nrightarrow K, \\ \prod_{g \in I} i_{g}^{*} u & \text { if } K=G^{\prime},\end{cases}
$$

where $I$ is the set of cosets representative for $G^{\prime}$ in the normalizer $N_{G}\left(G^{\prime}\right)$, the notation $G^{\prime} \nrightarrow K$ means $G^{\prime}$ is not conjugate to a subgroup of $K$, and $i_{g}^{*}$ is the conjugation map by $g$.

Let $A=\bigoplus^{n} Z / p^{r_{i}}$ and $k^{*}(A) \cong \bigotimes_{k^{*}} k^{*}\left[\left[u_{i}\right]\right] /\left(\left[p^{r_{i}}\right]\left(u_{i}\right)\right)$. Define an element $e_{A} \in k^{*}(A)$ by

$$
e_{A}=\prod_{0 \neq\left(\lambda_{1}, \ldots, \lambda_{n}\right) \in A}\left(\left[\lambda_{1}\right]\left(u_{1}\right)+{ }_{k} \cdots+_{k}\left[\lambda_{n}\right]\left(u_{n}\right)\right) .
$$

The element is unique except for multiplying units. It is immediate that if $A^{\prime} \nsubseteq A$ then $e_{A} \mid A^{\prime}=0$.

Lemma 3.13 (Lemma 2.4 in [5]). Let $\#(k) \geq \operatorname{rank}_{p} G$ and $k^{0}=Z / p$. If $\left[N_{G}(A) ; A\right]=q h, q=p^{s}$ and $(p, h)=1$, then there is $v_{A} \in k^{*}(G)$ such that

$$
v_{A} \mid A^{\prime}= \begin{cases}0 & \text { if } A \nrightarrow A^{\prime}, \\ e_{A}^{q} & \text { if } A=A^{\prime}\end{cases}
$$

Moreover if $y \in k^{*}(A)$ is invariant under $N_{G}(A)$, then there is an $\alpha(y)$ in $k^{*}(G)$ with $\alpha(y) \mid A=y^{q} e_{A}^{q}$.

Proof. Set $z=N[A \hookrightarrow G]\left(1+e_{A}\right)$. Then from (3.11) and the property of $e_{A}$, we have

$$
\begin{aligned}
z \mid A & =\left(1+e_{A}\right)^{q h}=\left(1+e_{A}^{q}\right)^{h} \\
& =1+h e_{A}^{q}+\text { terms of higher degree. }
\end{aligned}
$$

Taking $1 / h$ times the homogeneous component of $z$ of degree $e_{A}^{q}$, we have $v_{A}$. By taking $z=N[A \hookrightarrow G]\left(1+e_{A} y\right)$ we have $\alpha(y)$. Q.E.D. 
Theorem 3.14. Let $\#(k) \geq \operatorname{rank}_{p} G$ and $k^{0}=Z / p$. Then $r$ in (3.1) is an F-isomorphism.

Proof. Given $0 \neq\left(\lambda_{1}, \ldots, \lambda_{m}\right)=p^{s}\left(\lambda_{1}^{\prime}, \ldots, \lambda_{m}^{\prime}\right), A=\bigoplus^{m} Z / p^{r_{i}}$ with $\lambda_{1}^{\prime} \neq$ $0 \bmod p$ for some $i$, take $M$ to be a matrix such that the 1 st column is $\left(\lambda_{1}^{\prime}, \ldots, \lambda_{m}^{\prime}\right)$ and $M$ induces an automorphism of $A$. Then from (2.4) the kernel of the map in $k^{*}$-theory induced from

$$
Z / p^{s} \times Z / p^{r_{2}} \times \cdots \times Z / p^{r_{m}} \hookrightarrow A \stackrel{M^{-1}}{\rightarrow} A
$$

is the ideal generated by the following element in $k^{*}(A)$ :

$$
\left[\lambda_{1}\right]\left(\mu_{1}\right)+{ }_{k} \cdots+_{k}\left[\lambda_{m}\right]\left(u_{m}\right) .
$$

Therefore if $x \in k^{*}(A)$ satisfies $x \mid A^{\prime}=0$ for all $A^{\prime} \varsubsetneqq A$, then $x^{p^{r}} \in$ Ideal $e_{A}$.

Given $x \in \operatorname{Lim}$ inv $k^{*}(A)$ in (3.1), there is an abelian group $A$ such that $x \mid A^{\prime}=0$ for all $A^{\prime} \varsubsetneqq A$ and $0 \neq x \mid A \in k^{*}(A)^{N_{G}(A)}$. Then $x^{p^{s}} \mid A=e_{A} \alpha$ and $\alpha \in k^{*}(A)^{N_{G}(A)}$ since $e_{A} \in k^{*}(A)^{\operatorname{Aut}(A)}$ from the definition of $e_{A}$. By Lemma 3.13, we have completed the proof. Q.E.D.

\section{Relation to $H^{*}(G ; Z / p)$}

In [11], we see that when $G$ is an abelian $p$-group or $|G|=p^{3}$, there is an isomorphism

$$
\left(\mathrm{BP}^{*}(G) \otimes_{\mathrm{BP}^{*}} Z_{(p)}\right) / \sqrt{0} \cong H^{*}(G) / \sqrt{0} .
$$

We consider some extensions of this fact. Restriction maps to elementary abelian $p$-groups $e A$ of $G$ induce the map

$$
k^{*}(G) \stackrel{r^{\prime}}{\rightarrow} \operatorname{Liminv} k^{*}(e A) \stackrel{j}{\hookrightarrow} \prod k^{*}(e A)
$$

$e A \hookrightarrow G$, conjugacy classes of elementary abelian $p$-groups. Let

$$
J=\left(\text { ir }^{\prime}\right)^{-1}\left(\operatorname{Ideal}\left(p, v_{1}, \ldots\right)\right) .
$$

Of course, $k^{*}(G) / J$ is a quotient algebra of $k^{*}(G) \otimes_{k^{*}} Z / p$.

Theorem 4.2. If \# $(k) \geq \operatorname{rank}_{p} G$, then there is an $F$-isomorphism $\rho / J: k^{*}(G) / J$ $\hookrightarrow H^{*}(G ; Z / p) / \sqrt{0}$.

Proof. By the definition of $J$, there is an injection

$$
k^{*}(G) / J \hookrightarrow \prod k^{*}(e A) \otimes_{k^{*}} Z / p \cong \prod H^{*}(e A ; Z / p) / \sqrt{0} .
$$

Hence $k^{*}(G) / J \rightarrow_{\rho r^{\prime} / J} \operatorname{Liminv} H^{*}(e A ; Z / p) / \sqrt{0}$ is injective. By the same arguments as in the proof of Theorem 3.14, $r^{\prime}$ is $F$-epic. We show

$$
\rho: k^{*}(e A)^{W_{G}(A)} \rightarrow H^{*}(e A ; Z / p)^{W_{G}(A)}
$$


is also $F$-epic. Indeed, given $w \in W_{G}(A)$ and $\alpha \in H^{*}(e A ; Z / p)^{\langle w\rangle},|w|=$ $p^{k} p^{\prime},\left(p, p^{\prime}\right)=1$, we take $\tilde{\alpha} \in k^{*}(e A)$ with $\rho(\tilde{\alpha})=\alpha$ and

$$
\tilde{\alpha}_{w}=\frac{1}{p^{\prime}} \sum_{j=0}^{p^{\prime}-1} w^{*^{j p^{k}}}\left(\prod_{i=0}^{p^{k}-1} w^{*^{i p^{\prime}}} \tilde{\alpha}\right)
$$

so that $w^{*} \tilde{\alpha}_{w}=\tilde{\alpha}_{w}$ and $\rho\left(\tilde{\alpha}_{w}\right)=\alpha^{p^{r}}$. Therefore we can prove $\rho r^{\prime} / J$ is an $F$-isomorphism by the arguments similar to Theorem 3.14 .

Quillen's main theorem [6] says that $H^{*}(G ; Z / p) \rightarrow \operatorname{liminv} H^{*}(e A ; Z / p)$ is an $F$-isomorphism. Hence we have the theorem. Q.E.D.

Corollary 4.3. There is an F-isomorphism

$$
\rho: k(n)^{*}(G) \otimes_{k(n)^{*}} Z / p \hookrightarrow H^{*}(G ; Z / p) .
$$

Proof. Since there is cohomology theory $k^{*}$ such that $\rho: k^{*}(-) \rightarrow k(n)^{*}(-)$ and $\#(k)=\infty$, the map is $F$-epic from Theorem 4.2. By the Sullivan-Bockstein exact sequence, it is also injective. Q.E.D.

\section{Relation to the Morava $K$-theory}

Recall $P(n)^{*}=\mathrm{BP}^{*} /\left(p, v_{1}, \ldots, v_{n-1}\right) \cong Z / p\left[v_{n}, \ldots\right]$. We see in [11] that when $G$ is an abelian $p$-group or $|G|=p^{3}$, there is an isomorphism

$$
\mathrm{BP}^{*}(G) \otimes_{\mathrm{BP}^{*}} P(n)^{*} \cong P(n)^{*}(G) \text { for all } n \geq 1 \text {. }
$$

By the Sullivan-Bockstein exact sequence, $(5.1)$ is equivalent to

$$
v_{n}: \mathrm{BP}^{*}(G) \otimes_{\mathrm{BP}^{*}} P(n)^{*} \rightarrow \mathrm{BP}^{*}(G) \otimes_{\mathrm{BP}^{*}} P(n)^{*}
$$

is injective for each $n \geq 0$.

The Landweber exact functor theorem [4] says that if $\operatorname{BP}^{*}(G)$ satisfies (5.2), then $\mathrm{BP}^{*}(G) \otimes_{\mathrm{BP}^{*}}-$ is an exact functor for finite $\mathrm{BP}^{*}(\mathrm{BP})$ modules. Moreover, $P(n)^{*}(G) \otimes_{P(n)^{*}}-$ is also an exact functor from [12].

Theorem 5.3. If a p-Sylow subgroup $P$ of $G$ is a direct product of groups which satisfy (5.2), then we have

$$
\begin{aligned}
& \mathrm{BP}^{*}(G) \otimes_{\mathrm{BP}^{*}} P(n)^{*} \cong P(n)^{*}(G), \\
& \mathrm{BP}^{*}(G) \otimes_{\mathrm{BP}^{*}} K(n)^{*} \cong K(n)^{*}(G) .
\end{aligned}
$$

Proof. Let $P=P_{1} \oplus \cdots \oplus P_{s}$ and $P_{i}$ satisfies (5.2). By the exact functor theorem for $P(n)^{*}$-theory

$$
P(n)^{*}\left(-\wedge \mathrm{BP}_{i}\right) \cong P(n)^{*}(-) \otimes_{P(n)^{*}} P(n)^{*}\left(P_{i}\right)
$$

because both are cohomology theories with the same coefficient. Hence $P(n)^{*}(P)$ $\cong \bigotimes_{P(n)^{*}} P(n)^{*}\left(P_{i}\right)$. Therefore we have

$$
P(n)^{*}(P) \cong\left(\bigotimes_{\mathrm{BP}^{*}} \mathrm{BP}^{*}\left(P_{i}\right)\right) \otimes_{\mathrm{BP}^{*}} P(n)^{*} \cong \mathrm{BP}^{*}(P) \otimes_{\mathrm{BP}^{*}} P(n)^{*} .
$$


Hence $P$ satisfies (5.1) and so (5.2). Since $P(n)^{*}(G) \hookrightarrow P(n)^{*}(P)$, multiplying $v_{n}$ in $P(n)^{*}(G)$ is injective. By the Conner-Floyd type theorem, $P(n)^{*}(-) \otimes_{P(n)^{*}} K(n)^{*} \cong K(n)^{*}(-)$, and we have the theorem. Q.E.D.

\section{Minimal NONABElian $p$-GRoups}

For an odd prime $p$, the minimal nonabelian $p$-groups are of two types (Redéi [9])

Type 1. $G_{1}=\left\langle a, b ; a^{p^{\prime \prime}}=b^{p^{\beta}}=1,[a, b]=a^{p^{\alpha-1}}\right\rangle$,

Type 2. $G_{2}=\left\langle a, b, c ; a^{p^{\alpha}}=b^{p^{\beta}}=c^{p}=1,[a, b]=c,[c, a]=[c, b]=1\right\rangle$.

When $p=2$, there is an isomorphism $G_{1}(\alpha=2, \beta=1) \cong G_{2} \quad(\alpha=1, \beta=$ 1) and we need to add another type

$$
Q=\left\langle a, b ; a^{4}=1, a^{2}=b^{2}=[a, b]\right\rangle .
$$

For each of the above types, there is an exact sequence

$$
1 \rightarrow C \rightarrow G \rightarrow Z / p \oplus Z / p \rightarrow 1
$$

where $C$ is the center of $G$ and is isomorphic to $\left\langle a^{p}, b^{p}\right\rangle$ for Type 1 and $\left\langle c, a^{p}, b^{p}\right\rangle$ for Type 2. The induced Hochschild-Serre spectral sequence is

$$
\begin{aligned}
E_{2}^{*, *} & =H^{*}\left(Z / p \oplus Z / p ; \mathrm{BP}^{*}(C)\right) \\
& \cong \tilde{Z} / p\left[y_{1}, y_{2}\right] \otimes \bigwedge(\alpha) \otimes \operatorname{BP}^{*}(C) \Rightarrow \operatorname{BP}^{*}(G)
\end{aligned}
$$

where $\tilde{Z} / p[a]$ means $Z[a] /(p a)$ and $\left|y_{1}\right|=\left|y_{2}\right|=2$ and $|\alpha|=3$. Let us write

$$
\begin{aligned}
\mathrm{BP}^{*}(C) & =\mathrm{BP}^{*}\left[\left[u, u_{1}, u_{2}\right]\right] /\left([p](u),\left[p^{\alpha-1}\right]\left(u_{1}\right),\left[p^{\beta-1}\right]\left(u_{2}\right)\right) \text { or } \\
& =\mathrm{BP}^{*}\left[\left[u_{1}, u_{2}\right]\right] /\left(\left[p^{\alpha}\right]\left(u_{1}\right),\left[p^{\beta-1}\right]\left(u_{2}\right)\right) .
\end{aligned}
$$

We will compute the spectra sequence (6.2).

Type 2. Consider the quotient map $q$

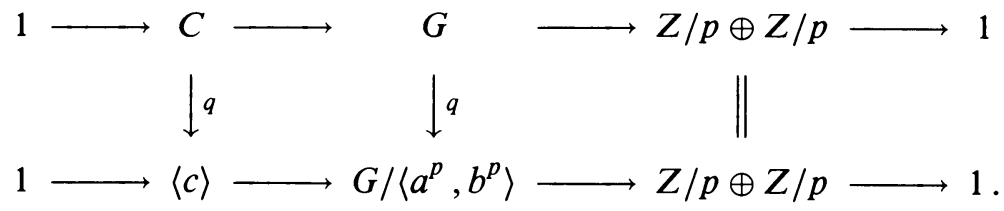

The spectral sequence $\widetilde{E}_{r}^{*, *}$ induced from the lower exact sequence is known from [11]. The differentials are

$$
\begin{gathered}
d_{3} u=\alpha, \\
d_{2 p-1} u^{p-1} \alpha=y_{1}^{p} y_{2}-y_{1} y_{2}^{p},
\end{gathered}
$$

and we get

$$
\begin{aligned}
\widetilde{E}_{2 p}^{*, *} \cong & \widetilde{E}_{\infty}^{*, *} \cong \mathrm{BP}^{*} \otimes\left(Z\left\{p u, \ldots, p u^{p-1}\right\}\right) \oplus Z / p\left[\left[y_{1}, y_{2}\right]\right] /\left(y_{1}^{p} y_{2}-y_{1} y_{2}^{p}\right) \\
& \otimes Z\left[\left[u^{p}\right]\right] /([p](u)) .
\end{aligned}
$$


There are splitting maps $\langle b\rangle \rightleftarrows G,\langle a\rangle \rightleftarrows G$ which induced that $u_{1}$ and $u_{2}$ are permanent cycles. Since $\mathrm{BP}^{*}\left(\left\langle a^{p}, b^{p}\right\rangle\right)$ is a flat $\mathrm{BP}^{*}$-module for $\mathrm{BP}^{*}(\mathrm{BP})$ modules, we have

$$
E_{r}^{*, *} \otimes_{\mathrm{BP}^{*}} \mathrm{BP}^{*}\left(\left\langle a^{p}, b^{p}\right\rangle\right) \cong E_{r}^{*, *} .
$$

In particular, we get

$$
E_{\infty}^{*, *} \cong(6.6) \otimes_{\mathrm{BP}^{*}} \mathrm{BP}^{*}\left[\left[u_{1}, u_{2}\right]\right] /\left(\left[p^{\alpha-1}\right]\left(u_{1}\right),\left[p^{\beta-1}\right]\left(u_{2}\right)\right) .
$$

Type 1 case. First, we consider the case $\beta=1$ and denote by $\widetilde{E}_{r}^{*, *}$ the induced spectral sequence from (6.1). Consider also the spectral sequence converging to $H^{*}\left(G_{1} ; Z\right)$. Since

$$
H^{2}\left(G_{1} ; Z\right) \cong \operatorname{Hom}\left(G_{1} ; Q / Z\right) \cong Z / p^{\alpha-1}, \quad \beta=1,
$$

we get $d_{3} u=\alpha$. Moreover, considering the spectral sequence converging to $H^{*}\left(G_{1} ; Z / p\right)$, we also have $d_{2 p-1} u^{p-1} \alpha=y_{1}^{p} y_{2}-y_{1} y_{2}^{p}$. Similar results hold in $\mathrm{BP}^{*}$-theory. Therefore $E_{\infty}^{*, *} \cong E_{2 p}^{*, *} \cong(6.6)$. The flatness of $\operatorname{BP}^{*}\left(\left\langle b^{p}\right\rangle\right)$ implies $E_{r}^{*, *} \cong(6.6) \otimes_{\mathrm{BP}^{*}} \mathrm{BP}^{*}\left(\left\langle b^{p}\right\rangle\right)$,

$$
E_{\infty}^{*, *} \cong(6.6) \otimes_{\mathrm{BP}^{*}} \mathrm{BP}^{*}\left[\left[u_{2}\right]\right] /\left(\left[p^{\beta-1}\right]\left(u_{2}\right)\right) .
$$

Corollary 6.10. For $G_{1}$ or $G_{2}$, the image of the map

$$
j: \mathrm{BP}^{*}(G) \rightarrow \mathrm{BP}^{*}(\langle c\rangle) \otimes_{\mathrm{BP}^{*}} Z / p \cong Z / p[u]
$$

is $\operatorname{Im} j=Z / p\left[u^{p}\right]$.

Remark 6.11. For $G_{2}, \alpha \geq 2, \beta \geq 2$, the image of the map

$$
j: H^{*}\left(G_{2}\right) \rightarrow H^{*}(\langle c\rangle) \cong \tilde{Z} / p[u]
$$

is $\operatorname{Im} j=\operatorname{Ideal} u^{2}$. (See $[14,19]$ for more details.) We explain here the difference of spectral sequences for $H^{*}\left(G_{2}\right)$ and $\operatorname{BP}^{*}\left(G_{2}\right)$. Consider the spectral sequence from (6.1) for $\alpha=2$ and $\beta=2$ :

$$
\begin{gathered}
E_{2}^{*, *}=H^{*}\left(Z / p \oplus Z / p ; H^{*}(C)\right) \Rightarrow H^{*}(G), \\
E_{2}^{*, *}(Z / p)=H^{*}\left(Z / p \oplus Z / p ; H^{*}(C ; Z / p)\right) \Rightarrow H^{*}(G ; Z / p) .
\end{gathered}
$$

Then $E_{2}^{*, 0}(Z / p)=Z / p\left[y_{1}, y_{2}\right] \otimes \wedge\left(x_{1}, x_{2}\right), E_{2}^{0, *}(Z / p)=Z / p\left[u_{1}, u_{2}, u_{3}\right] \otimes$ $\bigwedge\left(z_{1}, z_{2}, z_{3}\right)$, and $E_{2}^{*, *^{\prime}}(Z / p) \cong E_{2}^{*, 0}(Z / p) \otimes E_{2}^{0, *^{\prime}}(Z / p)$. The integral parts are $E_{2}^{*, 0} \cong \widetilde{Z} / p\left[y_{1}, y_{2}\right] \otimes \Lambda\left(\beta\left(x_{1} x_{2}\right)\right)$ where $\beta$ is the Bockstein operation, $E_{2}^{0, *} \cong$ $\tilde{Z} / p\left[u_{1}, u_{2}, u_{3}\right] \otimes\left\{1, \beta\left(z_{i} z_{j}\right), \beta\left(z_{1} z_{2} z_{3}\right)\right\}$, and $E_{2}^{*, *^{\prime}} \cong E_{2}^{*, 0} \otimes E_{2}^{0, *^{\prime}}(Z / p)$ for $*>0$. The first differentials are

$$
d_{2} z_{1}=y_{1}, \quad d_{2} z_{2}=y_{2}, \text { and } d_{2} z_{3}=x_{1} x_{2} .
$$

Then $d_{3} u_{3}=\beta\left(x_{1} x_{2}\right) \neq 0$. However, $d_{3} u_{3}^{2}=0$. Indeed,

$$
d_{2}\left(x_{2} \beta\left(z_{1} z_{3}\right)-x_{1} \beta\left(z_{2} z_{3}\right)\right)=\beta\left(x_{1} x_{2}\right) u_{3},
$$

which is also the image $d_{3} u_{3}^{2}$. Therefore we see that $u_{3}^{2}$ is a permanent cycle. Remark 6.12. From Corollary 6.10 and Remark 6.11, the map $\rho / J$ in Theorem 4.2 is not epic for $G_{2}, \beta \geq 2, \alpha \geq 2$. 
Theorem 6.13. If a p-Sylow subgroup $P$ of $G$ is a direct product of minimal nonabelian p-groups and abelian p-groups, then

$$
\begin{aligned}
& \mathrm{BP}^{*}(G) \otimes_{\mathrm{BP}^{*}} P(n)^{*} \cong P(n)^{*}(G), \\
& \mathrm{BP}^{*}(G) \otimes_{\mathrm{BP}^{*}} K(n)^{*} \cong K(n)^{*}(G) .
\end{aligned}
$$

Proof. From Theorem 5.3, we need to prove (5.1) only in the cases $G_{1}$ and $G_{2}$. We will prove here the case $G_{2}$ only; the other case is proved by a similar argument. Consider the exact sequence

$$
1 \rightarrow\left\langle a^{p}, b^{p}\right\rangle \rightarrow G_{2} \rightarrow E \rightarrow 1,
$$

where $E=G_{2}(\alpha=1, \beta=1)$, and the induced spectral sequences

$$
\begin{aligned}
& E_{2}^{*, *}=H^{*}\left(E ; P(n)^{*}\left(\left\langle a^{p}, b^{p}\right\rangle\right)\right) \cong H^{*}\left(E ; P(n)^{*}\right) \otimes_{P(n)^{*}} P(n)^{*}\left(\left\langle a^{p}, b^{p}\right\rangle\right) \\
& \Rightarrow P(n)^{*}\left(G_{2}\right), \\
& \widetilde{E}_{2}^{*, *}=H^{*}(E ; P(n)) \Rightarrow P(n)^{*}(E) .
\end{aligned}
$$

Since all elements in $P(n)^{*}\left(\left\langle a^{p}, b^{p}\right\rangle\right)$ are permanent, we have $E_{r}^{*, *} \cong \widetilde{E}_{r}^{*, *} \otimes_{P(n)^{*}}$ $P(n)^{*}\left(\left\langle a^{p}, b^{p}\right\rangle\right)$ by flatness and naturality. In particular, $\widetilde{E}_{\infty}^{*, *}$ is generated by even-dimensional elements; so is $E_{\infty}^{*, *}$. Hence $G_{2}$ satisfies $(5.2)$ because if $v_{n}: P(n-1)^{*}\left(G_{2}\right) \rightarrow P(n-1)^{*}\left(G_{2}\right)$ is not injective, then $P(n)^{\text {odd }}\left(G_{2}\right) \neq 0$ by the Sullivan-Bockstein spectral sequence. Q.E.D.

Theorem 6.14. If a p-Sylow subgroup $p$ of $G$ is a direct product of minimal nonabelian p-groups and abelian groups, then $r$ in (3.1) is injective for $k^{*}=\mathrm{BP}^{*}$ or $P(n)^{*}$.

Proof. We need to prove the case $G=G_{1}$ or $G_{2}$. We will prove the case $k^{*}=\mathrm{BP}^{*}$ and the other cases are proved similarly. Let $A$ be a maximal abelian $p$-subgroup of $G$. Let us denote by $E_{r}^{*, *}(A)$ the spectral sequence induced from

$$
1 \rightarrow C \rightarrow A \rightarrow Z / p \rightarrow 1 .
$$

Then the spectral sequence collapses and we have

$$
E_{\infty}^{*, *}(A) \cong \mathrm{BP}^{*}(C) \otimes \tilde{Z} / p[y] .
$$

Let us denote by $E_{r}^{*, *}(G)$ the spectral sequence induced from (6.1). Then we know $E_{\infty}^{*, *}(G)$ by $(6.6),(6.8)$, and (6.9).

We will prove that for each $x \in E_{\infty}^{*, *}(G)$, there is an abelian subgroup $A$ with $i^{*}(x) \neq 0$ in $E_{\infty}^{*, *}(A), i: A \hookrightarrow G$.

When $x \in E_{\infty}^{0, *}(G)$, this is obvious since $E_{\infty}^{0, *}(G) \hookrightarrow E_{\infty}^{0, *}(A)$.

We will prove that $i^{*}(x) \neq 0$ for $x \in E_{\infty}^{r, *}(G), r$ positive. Suppose $x$ is written such that $x=a y_{1}^{s}+y_{2} c$ where $a \neq 0$ in $\mathrm{BP}^{*} / p\left[\left[u^{p}\right]\right] /([p](u))$. Then $x \mid\langle a\rangle=a y_{1}^{s} \neq 0$. Suppose $x$ is written so that

$$
x=a y_{1}^{s}\left(\lambda_{1} y_{1}^{p-1} y_{2}+\lambda_{2} y_{1}^{n-2} y_{2}^{2}+\cdots+\lambda_{p-1} y_{1} y_{2}^{p-1}\right)+b\left(y_{1}^{s+p} y_{2}+\cdots\right)+\cdots .
$$


If $A / C \cong\left\langle a b^{\mu}\right\rangle$, we take a two-dimensional element $y$ so that $i^{*}\left(y_{1}\right)=y$ and $i^{*}\left(y_{2}\right)=\mu y$. Hence

$$
i^{*}(x)\left(\lambda_{1} \mu+\lambda_{2} \mu^{2}+\cdots+\lambda_{p-1} \mu^{p-1}\right) a y^{s+p}+b^{\prime} .
$$

Since $0=1-\mu^{p-1}=(1-\mu)\left(1+\mu+\cdots+\mu^{p-2}\right)$, if $i^{*}(x)=0$ for all $\mu \neq 1$, then $\lambda_{1}=\lambda_{2}=\cdots=\lambda_{p-1}=1$. But when $\mu=1, i^{*}(x)=(p-1) a y^{s+p}+b^{\prime} \neq 0$. Q.E.D.

Proposition 6.15. For each minimal nonabelian p-group $G$, the restriction maps $\mathrm{BP}^{*}(G) \rightarrow \mathrm{BP}^{*}(A)^{W_{G}(A)}$ are epic for all maximal abelian subgroups $A$.

Proof. Each maximal abelian subgroup of $G$ is isomorphic to $\left\langle a b^{\mu}, c, b^{p}\right\rangle$ or $\langle b, c\rangle$ (Type 1 case $c=a^{p}$ ). We will prove the map is epic for the case $A=\left\langle a, c, b^{p}\right\rangle$ and Type 2 . The other cases are proved similarly.

The map induced from the conjugation on $b$ is given by

$$
b^{*} u=u+_{\mathrm{BP}}\left[p^{\alpha-1}\right]\left(y_{1}\right), \quad b^{*} y_{1}=y_{1}, \quad b^{*} y_{2}=y_{2} .
$$

We can prove that

$$
\operatorname{BP}^{*}(A){ }^{\langle b\rangle} \cong \frac{\mathrm{BP}^{*}\left(\left\{1, N u, \ldots, N u^{p-1}\right\} \otimes\left[\left[U, y_{1}, y_{2}\right]\right]\right)}{\left([p](u),\left[p^{\alpha-1}\right]\left(y_{1}\right),\left[p^{\beta-1}\right]\left(y_{2}\right)\right)}
$$

where

$$
\begin{gathered}
N u^{s}=\sum b^{i *} u^{s}=p u^{s}+\cdots \\
U=\prod b^{i *} u=u^{p}+p^{\alpha-1} y_{1}^{p}+\cdots
\end{gathered}
$$

Therefore, from (6.6) and (6.8) we show the epimorphism.

The invariant is computed, for example, as follows. Let

$$
x=\left(u^{s}+a_{1} u^{s+1}+\cdots\right) y_{1}^{k}+b y_{1}^{k+1}+\cdots \quad \text { in } \operatorname{BP}^{*}(\langle a, c\rangle),
$$

with $s \neq 0 \bmod p$ and $a_{i} \in \mathrm{BP}^{*}[[y]] /\left[p^{\alpha x}\right](y)$. Then

$$
\begin{aligned}
b^{*} x= & \left(\left(u+_{\mathrm{BP}}\left[p^{\alpha-1}\right] y_{1}\right)^{s}+a_{1}\left(u+_{\mathrm{BP}}\left[p^{\alpha-1}\right] y_{1}\right)^{s+1}+\cdots\right) y_{1}^{k}+\cdots, \\
\left(1-b^{*}\right) x & \equiv p^{\alpha-1}\left(\left(s u^{s-1} y_{1}+v_{1} s u^{p+s-2} y_{1}+\cdots\right)+a_{1}(s+1) u^{s} y_{1}+\cdots\right) y_{1}^{k} \\
& \equiv s v_{1}^{\alpha-1} u^{(p-1)(\alpha-1)+s-1} y_{1}^{k+1} \bmod \left(p^{\alpha}, y_{1}^{k+2}, u^{(p-1)(\alpha-1)+s}\right),
\end{aligned}
$$

which is nonzero. Q.E.D.

Ravenel conjectured that $r$ in (3.1) is isomorphic for $k^{*}=$ BP. However this does not correct. Suppose $p \geq 3$ and $G=G_{2} \quad(\alpha=\beta=1)$. Let $A^{\mu}=\left\langle a b^{\mu}, c\right\rangle$ and $A^{p}=\langle b, c\rangle$ be the maximal abelian subgroups in $G$. By the arguments similar to the proof of Proposition 6.15, there is an element $\tilde{y}_{\mu} \in \mathrm{BP}^{2}\left(A^{\mu}\right)^{W_{G}\left(A_{\mu}\right)}$ such that $\tilde{y}_{\mu} \mid\left\langle a b^{\mu}\right\rangle \neq 0 \bmod \left(p, v_{1}, \ldots\right)$ and $\hat{y}_{\mu} \mid\langle c\rangle=0$. Consider the element

$$
y=\left(0, \tilde{y}_{1}, 0,0, \ldots, 0\right) \in \mathrm{BP}^{*}\left(A^{1}\right)^{W} \times \operatorname{BP}^{*}\left(A^{1}\right)^{W} \times \cdots \times \mathrm{BP}^{*}\left(A^{p}\right)^{W},
$$


which is in $\operatorname{Lim~BP}^{*}(A)$ since $A^{\mu} \cap A^{\lambda}=\langle c\rangle$ for $\mu \neq \lambda$. Recall that [11] $\mathrm{BP}^{*}(G) /\left(p, v_{1}, \ldots\right)$ is generated by $y_{1}$ and $y_{2}$ with $y_{1}\left|A^{0}=\tilde{y}_{0}, y_{1}\right| A^{p}=0$, $y_{2} \mid A^{0}=0$ and $y_{2} \mid A^{p}=\tilde{y}_{p}$. Hence there is no two-dimensional element $y$ in $\mathrm{BP}^{*}(G)$ such that $y \mid A^{1}=\tilde{y}_{1}$ and $y \mid A^{\mu}=0$ for all $\mu \neq 1$.

\section{APPLiCations; NONABELIAN $p$-SUBgROUP}

In this section we consider the existence of nonabelian $p$-subgroups of topological groups by using Corollary 6.10 .

Theorem 7.1. Let $G$ be a compact group such that $H^{*}(B G)_{(p)}$ is finitely generated as a ring and $\rho: \mathrm{BP}^{*}(B G) \rightarrow H^{*}(B G) /(p, \sqrt{0})$ is epic. If $G$ contains nonabelian p-subgroups, then there is a ring generator $x \in H^{*}(G) /(p, \sqrt{0})$ with $2 p|| x \mid$.

Proof. Let $P$ be a minimal nonabelian $p$-subgroup and $D \cong Z / p$ be the subgroup generated by $c$ for Type 2 and $a^{p^{n-1}}$ for Type 1. Consider the following commutative diagram:

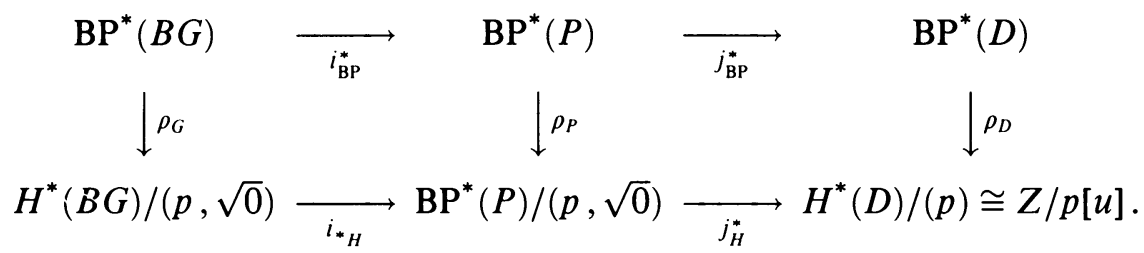

From Corollary 6.10, $\operatorname{Im}\left(\rho_{D} j_{\mathrm{BP}}\right)=Z / p\left[u^{p}\right]$. Hence

$$
\operatorname{Im}\left(j_{H}^{*} i_{H}^{*} \rho_{G}\right)=\operatorname{Im}\left(\rho_{D} j_{\mathrm{BP}}^{*} i_{\mathrm{BP}}^{*}\right) \subset Z / p\left[u^{p}\right] .
$$

Since $\rho_{G}$ is epic, $\operatorname{Im}\left(j_{H}^{*} i_{H}^{*}\right) \subset Z / p\left[u^{p}\right]$. From Quillen's main theorem of equivariant cohomology [6], $j_{H}^{*} i_{H}^{*} \neq 0$ for some $*>0$. Therefore there is a ring generator $x \in H^{*}(G) /(p, \sqrt{0})$ such that $j_{H}^{*} i_{H}^{*}(x)=u^{p s}$. Q.E.D.

Corollary 7.2. Let $G$ be a compact Lie group containing nonabelian p-subgroups.

(1) If $H^{*}(G)_{(p)} \cong \Lambda\left(x_{1}, \ldots, x_{n}\right)$, then there is $i$ with $2 p|| x_{i} \mid+1$.

(2) If $H^{*}(B G) /(p, \sqrt{0})$ is generated by $c_{i_{s}}, 1 \leq s \leq n$, $i_{s}$ th Chern classes of some representations, then there is $s$ such that $2 p \mid i_{s}$.

Remark 7.3. (1) of the above corollary is an immediate consequence of a result of Borel-Serre [15] and its converse also holds. Let $P \subset G$ be a $p$-group. By [15], we may (after conjugation) assume $P \subset N(T)$, the normalizer of a maximal torus $T$. If $P$ is nonabelian, then $P \not \subset T$ and $p$ divides the order $|W|$ of the Wyle group $W=N(T) / T$. Since $|W|=\prod\left(\left|x_{i}\right|+1\right) / 2$, we have (1).

Conversely, if $p|| W \mid$, then $N(T)$ contains nonabelian $p$-subgroups. The extension $T \rightarrow N(T) \rightarrow W$ defines an element of $H^{2}(W, T) \cong 0$ or $Z / 2 \oplus$ $\cdots \oplus Z / 2$ [18]. So an element of order $p$ in $W$ lifts to an element $x$ of order 
$p(, 2$ or 4 for $p=2)$. Let $V \subset T$ be the set of solutions of $t^{p}=1 \quad\left(t^{4}=1\right.$ for $p=2)$. Since $x$ acts nontrivially on $T, x$ also acts nontrivially on $V$. If we consider $V$ as a vector space over $F_{p}$, then the action on it is given by a Jordan decomposition, so we can find a subspace of dimension 2 on which the action is given by $\left(\begin{array}{ll}1 & 1 \\ 0 & 1\end{array}\right)$. This means that there is a subgroup of Type 2 $\alpha=\beta=1$ (nonabelian group of order $\leq 4^{3}$ for $p=2$ ).

This remark is due to J. F. Adams. The author is grateful to Professor Adams for his kind comments.

Example 7.4. The cohomologies of simply connected simple Lie groups are known and the cohomologies of some cases of their classifying spaces are known. For example,

$$
\begin{aligned}
& H^{*}(B S U(n)) \cong Z\left[y_{4}, \ldots, y_{2 n-1}\right], \\
& H^{*}\left(B E_{7}\right)_{(p)} \cong Z_{(p)}\left[y_{i} \mid i=4,12,16,20,24,28,36\right] \text { for } p \geq 5 .
\end{aligned}
$$

Thus $S U(n),(, \operatorname{Sp}(n), \mathrm{SO}(2 n+1))$ contains nonabelian $p$-subgroup if and only if $p \leq n$. The exceptional Lie group $G_{2}$ (resp. $F_{4}, E_{6}, E_{7}, E_{8}$ ) contains nonabelian $p$-subgroups if and only if $p \leq 3$ (resp. $\leq 3, \leq 5, \leq 7, \leq 7$ ).

Let $G\left(F_{q}\right)$ be the $F_{q}$-rational points of the universal Chevally group of the reductive complex Lie group type $G$. Let $q=p^{s}$ and $l \neq p$. Then $H^{*}(B G ; Z / l) \cong H^{*}\left(B G\left(\bar{F}_{p}\right) ; Z / l\right)$ where $\bar{F}_{p}$ is the algebraic closure of $F_{p}$ [16]. the cohomology of the $F_{q}$-rational points is computed by considering the coinvariant under the Frobenius-Adams operation $\sigma_{p}$. Let $r$ be the smallest number such that $q^{r}=1 \bmod l$. Quillen showed $H^{*}\left(\mathrm{GL}_{n}\left(F_{q}\right)\right) /(l, \sqrt{0}) \cong$ $Z / l\left[c_{r}, c_{2 r}, \ldots, c_{r[n / r]}\right]$; in this case we get $\sigma_{q} c_{i}=q^{i} c_{i}$. Hence if $\operatorname{GL}_{n}\left(F_{q}\right)$ contains nonabelian $l$-subgroups, then $l r \leq n$. Exceptional Lie group types are computed by Kleinerman [17]. For example, in the case $G=E_{7}, \sigma_{q} y_{i}=q^{i / 2} y_{i}$. Hence $H^{*}\left(B E_{7}\left(F_{q}\right)\right) /(l, \sqrt{0}) \cong Z / l\left[y_{i}|2 l| i\right]$. Therefore we see that if $E_{7}\left(F_{q}\right)$ contains nonabelian 5-subgroups (resp. 7-subgroups), then $r=1,2,5,10$ (resp. $r=1,2,7,14)$. When $l=p$, exceptional Lie types always contain nonabelian l-subgroups, since they contain $\mathrm{SL}_{3}\left(F_{p}\right)$.

\section{REFERENCES}

1. L. Evens, A generalization of the transfer map in the cohomology of groups, Trans. Amer. Math. Soc. 108 (1963), 54-65

2. D. Johnson and S. Wilson, BP operations and Morav's extraordinary K-theories, Math. Z. 144 (1975), 55-75.

3. P. Landweber Conference, flatness and cobordism of classifying spaces, Proc. Aarhus Summer Institute on Algebraic Topology 1970, pp. 256-269.

4. Homological properties of comodules over $M U_{*}(M U)$ and $\mathrm{BP}_{*}(\mathrm{BP})$, Amer. J. Math. 98 (1976), 591-610.

5. D. Quillen, A topological criterion for p-nilpotency, J. Pure Appl. Algebra 4 (1971), 373-376.

6. __ The spectrum of an equivariant ring. I, II, Ann. of Math. 94 (1971), 549-572, 573-602. 
7. _ Elementary proofs of some results of cobordism theory using Steenrod operation, Adv. in Math. 7 (1971), 29-56.

8. D. Ravenel, private communication.

9. L. Redéi, Das schiefe Product in der Gruppentheorie, Comment. Math. Helv. 20 (1947), 225264.

10. C. Stretch Stable cohomology and cobordism of abelian groups, Math. Proc. Cambridge Philos. Soc. 90 (1981), 273-278.

11. M. Tezuka and N. Yagita, Cohomology of finite groups and the Brown-Peterson cohomology, Lecture Notes in Math., vol. 1370, Springer.

12. N. Yagita, The exact functor theorem for $\mathrm{BP}^{*} / I_{n}$-theory, Proc. Japan Acad. 52 (1976), 1-3.

13. - On relations between Brown-Peterson cohomology and the ordinary $\bmod p$ cohomology theory, Kodai Math. J. 7 (1984), 273-285.

14. _ On the dimension of spheres whose product admits a free action by a non abelian p-group, Quart. J. Math. Oxford Ser. 36 (1985), 117-127.

15. A Borel and J. P. Serre, Sur certain sous-groupes des groupes de Lie compacts, Comment. Math. Helv. 27 (1953), 128-139.

16. E. Friedlander and G. Mislin, Cohomology of classifying spaces of complex Lie groups and related discrete groups, Comment. Math. Helv. 59 (1984), 347-361.

17. S. Kleinerman, The cohomology of Chevally groups of exceptional Lie type, Mem. Amer. Math. Soc. 268 (1982).

18. S. Ihara and T. Yokonuma, On the second cohomology groups (Shur-multipliers) of finite reflection groups, J. Fac. Sci. Univ. Tokyo Sect. 11 (1965), 155-171.

19. C. B. Thomas, Characteristic classes and the cohomology of finite group, Cambridge Univ. Press, 1987.

20. M. Hopkins N. Kuhn, and D. Ravenel, Generalized group characters and complex oriented cohomology theories (to appear).

Department of Mathematics, Musashi Institute of Technology, Tamazutumi SetaGaYA, TOKYO, JAPAN 